\title{
Bridge Engineering: Referees 2019
}

The following is a list of referees who have reviewed papers for Bridge Engineering between 1 December 2018 and 30 November 2019. The Institution of Civil Engineers is very grateful for their assistance.

We are continually looking for suitable reviewers for papers submitted to Bridge Engineering. Papers published in the Proceedings of the ICE must be submitted to at least two independent referees to judge accuracy, style, impact, importance and interest.

$\begin{array}{ll}\text { Aminuddin Ab Ghani } & \text { Kuo-Chun Chang } \\ \text { William Addis } & \text { Peter Clapham } \\ \text { Afaq Ahmad } & \text { Richard Clough } \\ \text { Ehsan Ahmadi } & \text { David Collings } \\ \text { Reza Akbari } & \text { Richard Cooke } \\ \text { Ahmet Emin Aktan } & \text { Robert Corbally } \\ \text { Pere Alfaras } & \text { Lee Cunningham } \\ \text { Tony Ambrose } & \text { Jeremy Cutter } \\ \text { Costas Anagnostopoulos } & \text { Luigi Di-Sarno } \\ \text { Chris Atkins } & \text { Marco Domaneschi } \\ \text { Hussein Aziz } & \text { James Dow } \\ \text { James Banks } & \text { Andy Foden } \\ \text { Saprava Bhattacharya } & \text { lan Frostick } \\ \text { Fabio Biondini } & \text { Fabio Gazzola } \\ \text { Simon Bourne } & \text { Graham Gedge } \\ \text { John Broomfield } & \text { Dave Gent } \\ \text { Philip Brown } & \text { Arndt Goldack } \\ \text { Michael Brown } & \text { Donya Hajializadeh } \\ \text { Andrea Caristo } & \text { Ciaran Hanley } \\ \text { Athol Carr } & \text { Chris Jackson } \\ \text { Joan Casas } & \text { Paul A. Jackson } \\ \text { Shengbo Chai } & \text { Stephen Jones } \\ \end{array}$

If you are interested in reviewing articles on any topic related to bridge engineering, please submit your name, qualifications or CV, and areas of expertise. We are in need of individuals who will agree to review papers in a timely fashion (within 3 to 4 weeks of receipt) and provide confidential feedback to the Editorial Advisory Panel concerning the quality of the paper and any suggested revisions that would be appropriate.

If you are such a person, please contact Kirsten Buchanan (tel.: +44 (0)207 665 2204; e-mail: kirsten.buchanan@icepublishing.uk) for more information on the referee process.

Juan Jorquera-Lucerga

Sivakumar Kandasam

Mohammad Kashani

Sameer Khan

Yail Jimmy Kim

Martin Kirk

Johann Kollegger

Manish Kumar

Ray Langley

Erik Larsen

Fang Li

Yongle Li

Brian Maddison

Barry Mawson

Padraig McCarron

John McElhinney

Luis Carlos Mendes

Stergios Mitoulis

Alan Monnickendam

Alan Mordey

Aogan Mulcahy

Wen-jie Niu
Camillo Nuti

Vikram Pakrashi

Artur Piekarczuk

John Place

Wan Qi-bai

Keith Ramsey

John Redpath

Ana M. Ruiz-Teran

Jessica Sandberg

Jose Santos

Mungo Stacy

Alfred Strauss

Riccardo Stroscio

Peter Takacs

Neil Tsang

Jose Turmo

Paolo Valvo

Daniel Vaz

Marco Vona

Yingliang Wang

Hoe-Chian Yeow 